\title{
FINITE RATE OF INNOVATION WITH NON-UNIFORM SAMPLES
}

\author{
Xiaoyao Wei $^{\star} \quad$ Thierry Blu $\quad$ Pier-Luigi Dragott ${ }^{\dagger}$ \\ ${ }^{\star}$ The Chinese University of Hong Kong, Hong Kong, China \\ $\dagger$ Imperial College, London, UK
}

\begin{abstract}
In this paper, we investigate the problem of retrieving the innovation parameters (time and amplitude) of a stream of Diracs from non-uniform samples taken with a novel kernel (a hyperbolic secant). We devise a non-iterative, exact algorithm that allows perfect reconstruction of $2 K$ innovations from as few as $2 K$ non-uniform samples. We also investigate noise issues and compute the Cramér-Rao lower bounds for this problem. A simple total least-squares extension of the algorithm proves to be efficient in reconstructing the location of a single Dirac from noisy measurements.
\end{abstract}

Index Terms - Signal sampling, finite rate of innovation, non-uniform, hyperbolic secant function, Cramér-Rao Bounds

\section{INTRODUCTION}

Sampling non-bandlimited — and in particular, sparse — signals has been a hot topic in recent years (see for example the special issue [1] and the papers therein). One approach is to modelize such signals using a finite number of parameters - a.k.a innovations - per unit of time, hence their name: "finite rate of innovation" (FRI) signals [2]. Examples of such signals include streams of Diracs, piecewise polynomials and piecewise sinusoidals. Very efficient SVD-based algorithms exist when the sampling kernels are periodized sinc kernels [3], modulated Gaussians [4], Strang-Fix kernels [5, 6] and even Cauchy-like analytic kernels [7].

However, one of the constraints of the FRI algorithms to date has been that the samples of the non-bandlimited signal have to be uniform, in order to achieve perfect reconstruction. Our contribution in this paper is twofold:

1. We present a new sampling kernel, the hyperbolic secant, that allows to sample and perfectly reconstruct streams of Diracs with a noniterative algorithm.

2. We demonstrate the possibility to perform non-uniform sampling with the new hyperbolic secant kernel, while

Xiaoyao Wei was supported by the Prof. Charles K. Kao Research Exchange Scheme. Thierry Blu was supported in part by an RGC grant \#CUHK410110 of the Hong Kong University Grant Council. Pier Luigi Dragotti was in part supported by the European Research Council (ERC) starting investigator award Nr. 277800 (RecoSamp). still being able to perform exact reconstruction using a non-iterative algorithm.

More specifically, from a stream of $K$ Dirac impulses with amplitudes $x_{k}$ and located at $t_{k}, k=1,2, \ldots, K$, we consider the set of $N$ arbitrary samples at $t=\tau_{n}$

$$
y_{n}=\sum_{k=1}^{K} x_{k} \varphi\left(\tau_{n}-t_{k}\right)
$$

where $\varphi(t)$ is the sampling kernel, and propose a novel reconstruction algorithm in the case where this kernel is the hyperbolic secant:

$$
\varphi(t)=\frac{1}{\cosh (a t)}, \quad \text { where } a>0 .
$$

Problems involving level-crossing A/D converters [8] or importance sampling [9] may benefit from these new algorithms. In general, this theory may have implications in all the domains where non-uniform sampling occurs such as for example geophysics, biomedical imaging and communications. For a review on the canonical non-uniform sampling problem and its applications, we refer to [10].

The outline of the paper is as follows. In Section 2 we develop the algorithm that enables the reconstruction from nonuniform measurements. In Section 3, we discuss the noise issues and present an algorithm to deal with it. We show simulation results that evaluate the performance of our algorithm in the presence of noise in Section 4 and finally conclude in Section 5 .

\section{SAMPLING SIGNALS WITH FRI}

By replacing the expression of the hyperbolic secant (2) in (1) we find that

$$
\begin{aligned}
y_{n} & =\sum_{k=1}^{K} \frac{x_{k}}{\cosh \left(a \tau_{n}-a t_{k}\right)} \text { for } n=1,2, \ldots, N \\
& =\frac{\mathrm{e}^{a \tau_{n}} P\left(\mathrm{e}^{2 a \tau_{n}}\right)}{Q\left(\mathrm{e}^{2 a \tau_{n}}\right)}
\end{aligned}
$$

where $P(x)$ and $Q(x)$ are polynomials of degree $K-1$ and $K$, respectively, defined by $P(x)=\sum_{k=0}^{K-1} p_{k} x^{k}$ and $Q(x)=$ 
$\sum_{k=0}^{K} q_{k} x^{k}$. Moreover, $Q(x)$ contains the information of the Dirac locations through the factorization

$$
Q(x)=\prod_{k=1}^{K}\left(1+\mathrm{e}^{-2 a t_{k}} x\right) .
$$

By multiplying both sides of (3) by $Q(x)$, we observe that the coefficients of $P$ and $Q$ satisfy the following linear system of equations:

$$
\sum_{k=0}^{K} q_{k} \mathrm{e}^{2 a \tau_{n} k} y_{n}=\sum_{k=0}^{K-1} p_{k} \mathrm{e}^{a \tau_{n}(2 k+1)}
$$

for $n=1,2, \ldots, N$. This system can be expressed in matrix/vector form as follows:

$$
\begin{aligned}
& \underbrace{\left[\begin{array}{cccc}
y_{1} & 0 & \cdots & 0 \\
0 & y_{2} & \cdots & 0 \\
0 & 0 & \ddots & 0 \\
0 & 0 & \cdots & y_{N}
\end{array}\right]}_{\boldsymbol{\Delta}_{N}} \underbrace{\left[\begin{array}{cccc}
1 & \mathrm{e}^{2 a \tau_{1}} & \cdots & \mathrm{e}^{2 K a \tau_{1}} \\
1 & \mathrm{e}^{2 a \tau_{2}} & \cdots & \mathrm{e}^{2 K a \tau_{2}} \\
\vdots & \vdots & \ddots & \vdots \\
1 & \mathrm{e}^{2 a \tau_{N}} & \cdots & \mathrm{e}^{2 K a \tau_{N}}
\end{array}\right]}_{\mathbf{V}_{K+1}} \underbrace{\left(\begin{array}{c}
q_{0} \\
q_{1} \\
\vdots \\
q_{K}
\end{array}\right)}_{\mathbf{q}} \\
& =\underbrace{\left[\begin{array}{cccc}
\mathrm{e}^{a \tau_{1}} & \mathrm{e}^{3 a \tau_{1}} & \cdots & \mathrm{e}^{a \tau_{1} \times(2 K-1)} \\
\mathrm{e}^{a \tau_{2}} & \mathrm{e}^{3 a \tau_{2}} & \cdots & \mathrm{e}^{a \tau_{2} \times(2 K-1)} \\
\vdots & \vdots & \ddots & \vdots \\
\mathrm{e}^{a \tau_{N}} & \mathrm{e}^{3 a \tau_{N}} & \cdots & \mathrm{e}^{a \tau_{N} \times(2 K-1)}
\end{array}\right]}_{\mathbf{W}_{K}} \underbrace{\left(\begin{array}{c}
p_{0} \\
p_{1} \\
\vdots \\
p_{K-1}
\end{array}\right)}_{\mathbf{p}}
\end{aligned}
$$

The system of $N$ equations $(N \geq 2 K)$ can be solved for the $2 K$ unknowns (up to a multiplicative constant). From its coefficients, we compute the roots, $z_{k}$, of $Q(x)$, from which we retrieve the Dirac locations, according to

$$
t_{k}=\frac{\ln \left(-z_{k}\right)}{2 a} .
$$

Having the locations $t_{k}$, the amplitude $x_{k}$ can be obtained by minimizing the mean square difference between the parametric FRI model and the samples, which once again amounts to solving a linear system of equations. We thus conclude that perfect reconstruction of Diracs is possible with the hyperbolic secant kernel and using only $N \geq 2 K$ samples. Reconstruction using samples at the critical sampling rate is exemplified in Figure 1.

\section{RECONSTRUCTION SCHEME IN THE PRESENCE OF NOISE}

The previous section demonstrates an ideal situation where no noise exists. However, in practice, any acquisition device introduces noise during the acquisition process. Hence we are looking for a reconstruction algorithm that can estimate the innovation parameters from the noisy samples:

$$
\hat{y}_{n}=\sum_{k=1}^{K} x_{k} \varphi\left(\tau_{n}-t_{k}\right)+\epsilon_{n}
$$

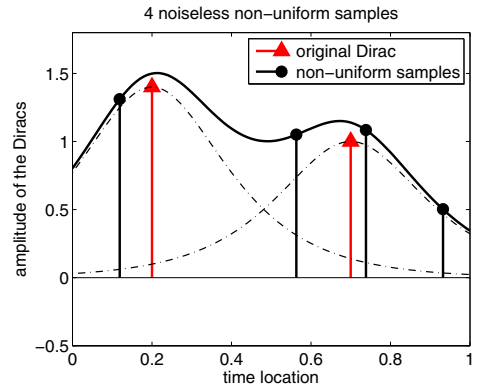

(a)

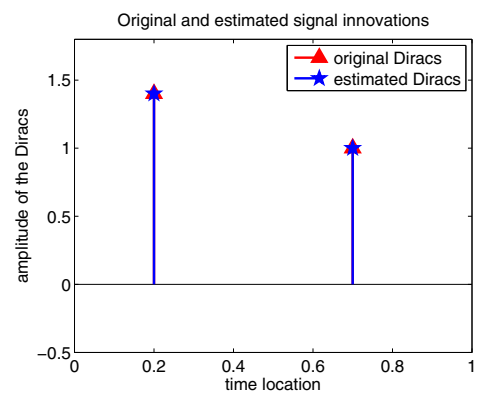

(b)

(6) Fig. 1. Retrieval of $K=2$ Diracs from $N=4$ nonuniform samples. Fig. 1(a) shows the two Diracs, the 4 non uniform samples, the signal $\sum_{k=1}^{K} x_{k} \varphi\left(\tau-t_{k}\right)$ where $\varphi(t)$ is the secant kernel (continuous line) and the two functions $x_{1} \varphi\left(\tau-t_{1}\right)$ and $x_{2} \varphi\left(\tau-t_{2}\right)$ (dashed lines). Fig. 1(b): original and reconstructed Diracs.

where the noise may be, for instance, additive Gaussian with zero mean and variance $\sigma^{2}$. To combat the noise, we propose a variation of the algorithm used to solve for $t_{k}$ in order to make the scheme more robust.

Given that the $\tau_{n}$ are sorted in increasing order of value, we notice that the entries in the two matrices $\mathbf{V}_{K+1}$ and $\mathbf{W}_{K}$ increase when going from top left to bottom right and these entries will increase more rapidly for large values of $a$ (recall that $a$ depends on the sampling kernel as indicated in Eq. (2)). This results in a large dynamics of matrix coefficients, which is likely to impair the accuracy of the computations. To alleviate this problem, both sides of the linear system (6) are multiplied by the following weighting matrix

$$
\mathbf{M}=\left[\begin{array}{cccc}
\mathrm{e}^{-K a \tau_{1}} & 0 & \cdots & 0 \\
0 & e^{-K a \tau_{2}} & \cdots & 0 \\
0 & 0 & \ddots & 0 \\
0 & 0 & \cdots & e^{-K a \tau_{N}}
\end{array}\right]
$$

Note that this practice does not really eradicate the problem, but improves the decomposition accuracy a bit. In other words, it provides similar decomposing accuracy for a comparatively larger $a$.

To further improve stability we propose the following ap- 
proach. First of all, the following relation holds:

$$
\boldsymbol{\Delta}_{N} \mathbf{V}_{K+1}^{\prime} \mathbf{q}=\mathbf{W}_{K}^{\prime} \mathbf{p}
$$

where $\mathbf{V}_{K+1}^{\prime}=\mathbf{M} \mathbf{V}_{K+1}$ and $\mathbf{W}_{K+1}^{\prime}=\mathbf{M} \mathbf{W}_{K+1}$.

Next, we eliminate the right hand side of Equation (10) by looking for an orthonormal $(N-K) \times N$ matrix $\mathbf{U}_{N-K}$ such that $\mathbf{U}_{N-K}^{T} \mathbf{W}_{K}^{\prime}=0 .{ }^{1}$ In this way we obtain the following new relation:

$$
\mathbf{U}_{N-K}^{T} \boldsymbol{\Delta}_{N} \mathbf{V}_{K+1}^{\prime} \mathbf{q}=\mathbf{0} .
$$

This is the annihilation equation that the polynomial $Q(x)$ satisfies and which is familiar to other FRI schemes. After QR decomposition of $\mathbf{V}_{K+1}^{\prime}$ into $\mathbf{Q}_{K+1} \mathbf{R}$, the above relation becomes

$$
\underbrace{\mathbf{U}_{N-K}^{T} \boldsymbol{\Delta}_{N} \mathbf{Q}_{K+1}}_{\mathbf{A}} \mathbf{R q}=\mathbf{0} .
$$

Notice that now both $\mathbf{U}_{N-K}$ and $\mathbf{Q}_{K+1}$ are orthonormal matrices and this provides the stability in the computations that we were looking for. In the presence of noise, however, (12) is not satisfied exactly. Nevertheless, a good estimation of Rq can be obtained by looking for a solution that minimizes $\|\mathbf{A R q}\|$ under the constrain that $\|\mathbf{R q}\|^{2}=1$. It is known that this minimization can be solved by performing Singular Value Decomposition (SVD) of A.

The eigenvector corresponding to the smallest eigenvalue given by this SVD procedure represents the desired estimation of Rq. Thus $\mathbf{q}$ can be obtained by $\mathbf{R}^{-1} \mathbf{R q}$. Finally, given $\mathbf{q}$, we follow the steps indicated in Section 2 to reconstruct the signal. More precisely, we can get the roots $z_{k}$ of $Q(x)$ to then estimate the innovation instants $t_{k}$ from (7).

\section{SIMULATION RESULTS}

Cramér-Rao lower bounds indicate the best possible performance for this parameter estimation problem. Hence the performance of the proposing method can be evaluated by checking the gap between the solution and the theoretical minimum.

\subsection{Cramér-Rao Lower Bounds}

The noisy samples (8) can be re-expressed as:

$$
\hat{y}_{n}=f(\boldsymbol{\Theta}, n)+\epsilon_{n}
$$

where $\boldsymbol{\Theta}=\left[x_{1}, x_{2}, \ldots, x_{K}, t_{1}, t_{2}, \ldots, t_{K}\right]^{T}$ is a vector of parameters to estimate, $n=1, \ldots, N$ and $f(\boldsymbol{\Theta}, n)=$ $\sum_{k=1}^{K} x_{k} \varphi\left(\tau_{n}-t_{k}\right)$.

Since any unbiased estimate of the unknown parameters $\Theta$ has a covariance matrix that is lower bounded by the inverse of the Fisher information matrix which is in this case

\footnotetext{
${ }^{1}$ This can be achieved, for example, by performing the QR decomposition of $\mathbf{W}_{K}^{\prime}$.
}

given by $[11,3]$ :

$$
\operatorname{CRB}(\boldsymbol{\Theta})=\sigma^{2}\left(\sum_{n=1}^{N} \nabla f(\boldsymbol{\Theta}, n) \nabla f(\boldsymbol{\Theta}, n)^{T}\right)^{-1} .
$$

We are therefore able to evaluate numerically the CR bounds using the above equation.

\subsection{Simulation Results}

We first show in Figure 2 an example of the noiseless and noisy samples (PSNR $=15 \mathrm{~dB}$, where PSNR is the peak signal-to-noise ratio defined as $10 \log _{10} \frac{\left|x_{1}\right|^{2}}{\sigma^{2}}$ ) that we obtain for $N=20$ and for two different choices of $a$. We have then implemented the algorithm proposed in Section 3 and show the results in Figure 3 and 4.

In Figure 3, we use $N=50$ samples to retrieve a single Dirac located at $t=0.5 \mathrm{sec}$. In this simulation, we have 100 realizations for each PSNR. The scatter plot of Figure 3

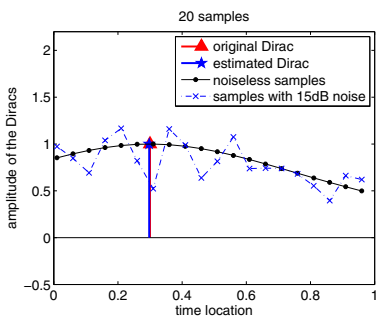

(a) $a=2$

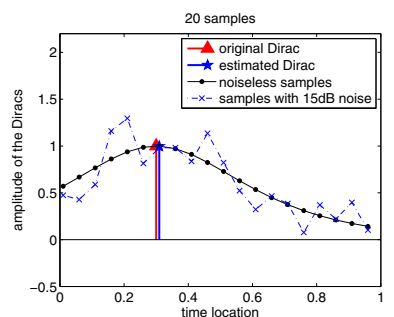

(b) $a=4$
Fig. 2. Noisy and noiseless samples for two different hyperbolic secant kernels.

indicates that the estimation performance is satisfactory up to $15 \mathrm{~dB}$ for a proper $a$ since the uncertainty on the location is quite close to the Cramér-Rao lower bound (see also Fig. 4 (d)).

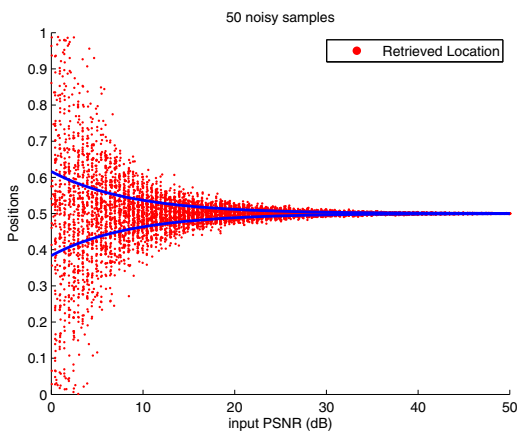

Fig. 3. Scatter plot of the Dirac's location from $N=50$ samples with $a=3$. See also Figure 4 (d).

In Figures 4, we compare the standard deviation of our estimator with the CR bounds and this for different numbers of 
samples and for different values of $a$. Performance improves with the number of samples and this in line with the improvement in the corresponding CR bound. We notice that there is a gap between our estimation performance and the optimal performance when $a$ is large. While the proposing algorithm cannot completely solve the problem of numerical issue arising from large $a$, the theory itself is tenable and works well for a proper $a$.

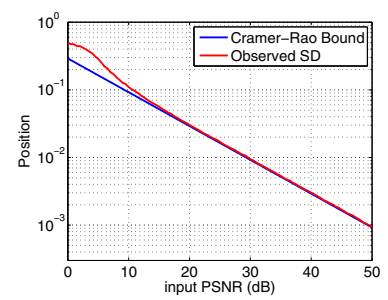

(a) $a=2,20$ samples

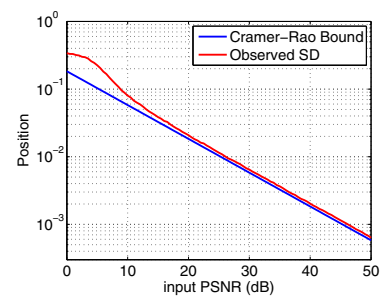

(c) $a=3$, 20 samples

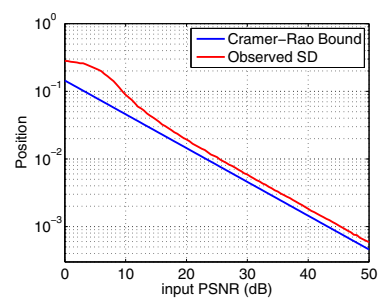

(e) $a=4,20$ samples

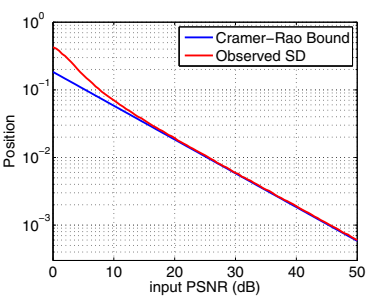

(b) $a=2,50$ samples

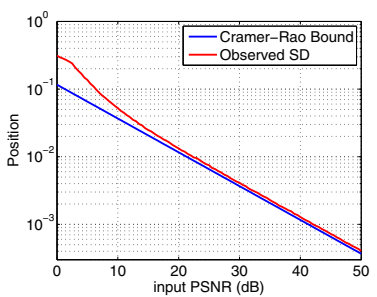

(d) $a=3,50$ samples

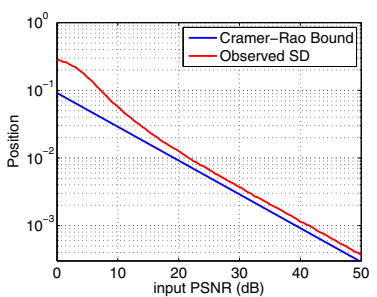

(f) $a=4,50$ samples
Fig. 4. Standard deviation of the proposed algorithm compared to Cramér-Rao bounds.

\section{CONCLUSIONS}

In this paper we have shown that it is possible to sample and perfectly reconstruct streams of Diracs using non-uniformly spaced samples. We have then presented a robust algorithm to reconstruct the Diracs when samples have been corrupted by noise. Preliminary results indicate that the algorithm performs well for medium to high SNRs. Future work will consider applying Cadzow iterative algorithm, as used in [3], to denoise the samples before retrieval of an FRI signal. We will also consider applying this sampling scheme to prospective applications.

\section{REFERENCES}

[1] R. Baraniuk, E. Candès, R. Nowak, and M. Vetterli, “Compressive sampling," IEEE Signal Processing Magazine, vol. 25(2), pp. 12-13, 2008.

[2] M. Vetterli, P. Marziliano, and T. Blu, "Sampling signals with finite rate of innovation," IEEE Transactions on Signal Processing, vol. 50, no. 6, pp. 1417-1428, 2002.

[3] T. Blu, P.L. Dragotti, M. Vetterli, P. Marziliano, and L. Coulot, "Sparse sampling of signal innovations," IEEE Signal Processing Magazine, vol. 25, no. 2, pp. 31-40, 2008.

[4] T. Blu, H. Bay, and M. Unser, "A new high-resolution processing method for the deconvolution of optical coherence tomography signals," in IEEE International Symposium on Biomedical Imaging: From Nano to Macro (ISBI 2002), 2002, pp. 777-780.

[5] P.L. Dragotti, M. Vetterli, and T. Blu, "Sampling moments and reconstructing signals of finite rate of innovation: Shannon meets Strang-Fix," IEEE Transactions on Signal Processing, vol. 55, no. 5, pp. 1741-1757, 2007.

[6] R. Tur, Y. Eldar, and Z. Friedman, "Innovation rate sampling of pulse streams with application to ultrasound imaging," IEEE Transactions on Signal Processing, vol. 59, no. 3, pp. 1827-1842, 2011.

[7] D. Kandaswamy, T. Blu, L. Spinelli, C. Michel, and D. Van De Ville, "Local multilayer analytic sensing for eeg source localization: Performance bounds and experimental results," in IEEE International Symposium on Biomedical Imaging: From Nano to Macro (ISBI 2011), 2011, pp. 479-483.

[8] K.M. Guan and A.C. Singer, "Opportunistic sampling by level-crossing," in IEEE International Conference on Acoustics, Speech and Signal Processing (ICASSP 2007), April 2007, vol. 3, pp. 1513-1516.

[9] H. Wang, S. Kay, and S. Saha, "An importance sampling maximum likelihood direction of arrival estimator,' IEEE Transactions on Signal Processing, vol. 56, no. 10, pp. 5082-5092, 2008.

[10] A. Aldroubi and K. Gröchenig, "Non-uniform sampling in shift-invariant spaces," SIAM Review, vol. 43, pp. 585-620, 2001.

[11] B. Porat and B. Friedlander, "Computation of the exact information matrix of Gaussian time series with stationary random components," IEEE Transactions on Acoustics, Speech and Signal Processing, vol. 34, no. 1, pp. 118-130, 1986. 